\title{
Analysis about Planning and Modification of the Upper and Lower Structures of the 38 Floor Apartment SkyHouse Project with Equivalent Static Analysis
}

\author{
Agus Fernando, Syahwandi, Resi Aseanto dan Agung Sumarno \\ Faculty of Engineering, Mercu Buana Univeristy ,Jakarta, Indonesia \\ Research Center of the SkyHouse AlamSutera Project, Tangerang, Indonesia \\ 41116320087@student.mercubuana.ac.id, wandi.syaf@yahoo.com
}

\begin{abstract}
The modeled building structure is a regular building, with the number of levels being varied. The structural model is divided into 38-level portals. This research uses the help of the SAP2000 v21 program to facilitate the earthquake analysis process. The results of the study that will be compared are displacements between levels and base shear that occur due to earthquake forces. The results of the analysis have shown that static analysis produces greater results for the structural models compared to dynamic analysis. The difference in displacement between levels produced by the two methods in the three structural models is still included in the displacement limits between levels of permission required in B. \& Wungo, (2012), so that the three models can still be analyzed by static analysis and dynamic analysis. Because the results of displacement and base shear in static analysis are greater than dynamic analysis, static analysis is safer if used for earthquake force loading in general structural calculations. Although in earthquake analysis, dynamic analysis is a more accurate analysis because the analysis process is closer to the actual situation.
\end{abstract}

Keywords:

Dynamic Analysis ,Static Analysis, SAP2000 v2, SNI 1726-2012.

\section{Introduction}

Indonesia is the fourth most populous country in the world with a population of around 269.6 million or $6 \%$ of the world's population. The large number of people in Indonesia causes the need for housing to increase. Therefore, there is a need for housing that does not require large land but can be used by many people, namely by the presence of high-rise buildings such as apartments.

The growth of apartments in Indonesia is increasing rapidly, the high demand for apartments in Indonesia is also due to land prices that are increasing from time to time. And this is in line with the increasing demand for housing for business people and professionals in the Alam Sutera areas. The type of housing chosen by the developer is an apartment with a residential concept that is in harmony with these conditions and is driven by regional conditions that can access entertainment centers, educational facilities and freeway facilities.

In planning this final project, it will focus on the discussion related to the comparison of the analysis of the modification of the upper and lower structures with earthquake static analysis in the planning of apartment buildings in the Alam Sutera area. This building has a 38-story building concept which must be taken into account against the loads acting on the building. The right method in analyzing earthquake loads, as well as planning shear walls that are able to withstand very large lateral forces and take into account the maximum forces that occur in structural elements both Columns, Shearwalls, Beams and Plates.

This study aims to compare the forces in the ultimate with the forces in the design and to determine whether the dimensions of the installed cross-section are able to withstand the forces in the ultimate due to the effects of external loading. Image of the structure of the SkyHouse AlamSutera Apartment project. Loading analysis refers to PBI 1983, and earthquake analysis uses equivalent static analysis method. Static analysis commonly used is the equivalent lateral force analysis. Dynamic analysis can be divided into two, namely analysis of variance response spectrum and analysis of seismic response history (time-history). Based on B. \& Wungo, (2012), there are many limitations given in the static equivalent analysis procedure. This limitation is made because the results of the equivalent static analysis are less precise for various types of structures being built. Therefore, dynamic analysis is needed to obtain a building response that is closer to the actual structure response when an earthquake occurs. The results of the calculation of the structure of both plates, beams, and columns show that the value of the design bending moment $(\mathrm{Mr})$ is greater than the ultimate bending moment $(\mathrm{Mu})$.

Determination of the lower structure system must be based on the following data:

1. Architectural plans including the required number of basement layers.

This work is licensed under a Creative Commons Attribution-ShareAlike 4.0 International License. 
2. The condition and situation of the surrounding buildings.

3. Results of soil investigations which include:

4. Ground water conditions.

5. Research pumping test if the basement bottom is below ground water mica.

6. The layer of soil supporting the building foundation.

7. Recommendations for the foundation system along with the carrying capacity and estimated settlement of the building.

The shape of the building and the structural system of the building frame are closely related to each other both horizontally and vertically. A structural system is called good. Several things that must be considered in determining the structural system against lateral loads include:

1. Diaphragm stiffness and structural rigidity

2. Force distribution and resistance concentration

3. Resistance on the outer perimeter (perimeter) of the building structure

4. Vertical plane jump (vertical setback)

5. Structural irregularity

6. There is a large torque without sufficient resistance to accommodate the torque

7. Short column effect

8. Discontinuity of structural strength and stiffness due to transfer girders, transfer floors or structural walls that are not continuous downwards, and structural walls that are located alternating both in the vertical and horizontal directions.

The concept of selecting a structural system and the concept of planning/designing the structure of the building, such as the configuration of the floor plan and the loading that has been adjusted to the basic requirements for planning a high-rise building that applies in Indonesia so that it is hoped that the results that will be obtained will not cause structural failure.

\section{Research Methodology}

In making an analysis, data is needed as a reference material. To be able to perform a good analysis, data that includes information and basic concepts related to the object to be analyzed is needed. The data can be classified into two types of data, namely primary data and secondary data..

Primary Data is data obtained from the field or survey results that can be directly used as a source in structural analysis. Material specification data is used to determine the characteristics of the materials used in the structure. Namely as follows:

a) Slab : f'c $=35 \mathrm{MPa}$

b) Beam : $\mathrm{f}^{\prime} \mathrm{c}=35 / 40 \mathrm{MPa}$

c) Column : $\mathrm{f}^{\prime} \mathrm{c}=35 / 40 / 45 / 50 \mathrm{MPa}$

d) Foundation: $\mathrm{f}^{\prime} \mathrm{c}=50 \mathrm{MPa}$

e) Reinforcement : fy $=400 \mathrm{MPa}$, for main reinforcement

fy $=240 \mathrm{MPa}$, for stirrup reinforcement

fy $=520 \mathrm{MPa}$, for strand reinforcement and PC-wire

Land Data Soil data obtained from the results of investigations and soil testing in the laboratory, consisting of :

1. Boring

2. Direct Shear Test

3. Liquid Plastic Limit Test

4. Sondir

5. Grain Size Accumulation Curve 
Figure 1. Final Project Compilation Flowchart

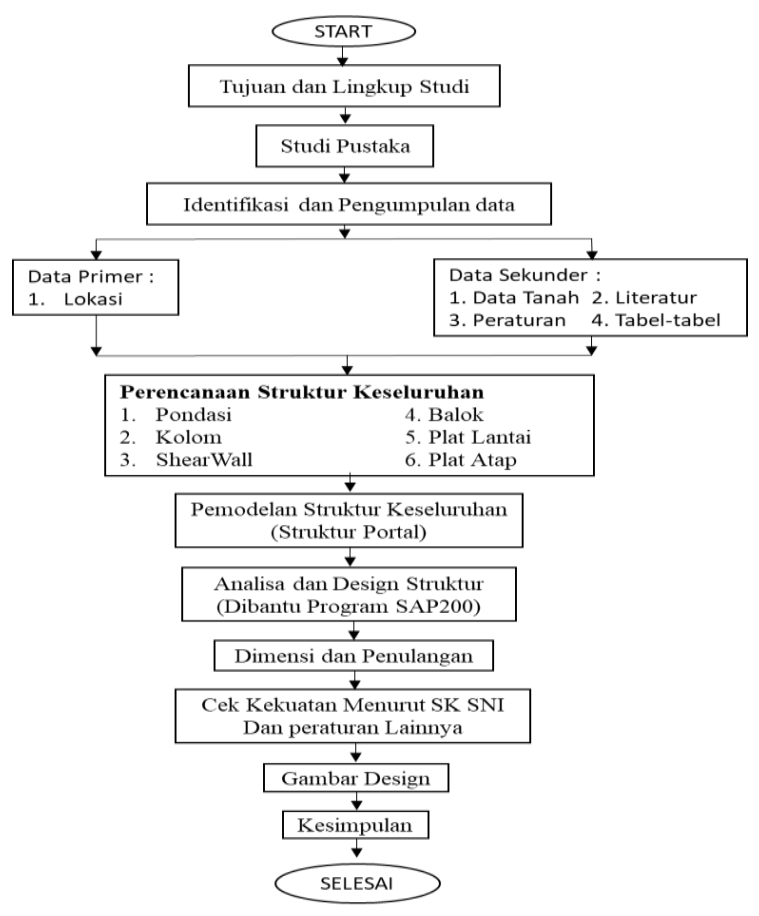

When modeling SAP 2000, the column elements will be released which will cause the moment in the bottom column to be 0 . This is done because the connection between the precast columns is not pure clamping so that the modeling in SAP 2000 is expected to be close to the actual behavior in the field. But for connection analysis is based on $\mathrm{P}$ axial column tension (derived from the moment when the connection is purely clamped) and shear. With the help of this computer program, the program output will be obtained in the form of internal forces acting on the structure due to all existing load combinations including earthquake loads. The flowchart for portal mechanics calculations using SAP 2000 :

Figure 2. SAP2000 Analysis Compilation Flowchart

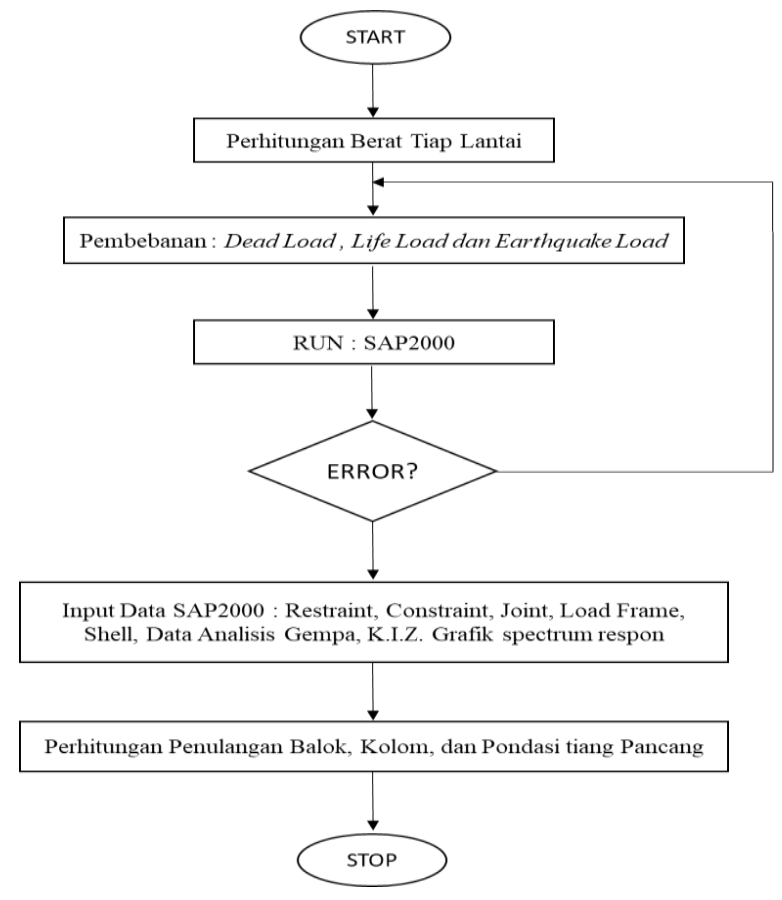

This work is licensed under a Creative Commons Attribution-ShareAlike 4.0 International License. 


\subsection{Slab Planning}

Slabs are reinforced concrete panels which may be reinforced in two or one direction depending on the structural system. If in the slab structure the ratio of long span to short span is 3, it will experience deflection in both axes directions. The slab load is carried in both directions by the four supporting beams around the slab panels, thus the slab becomes a slab that flexes in both directions. The slab planning steps are :

a) Determine boundary conditions, supports and span lengths.

b) Determine the working loads

c) Determine plate thickness

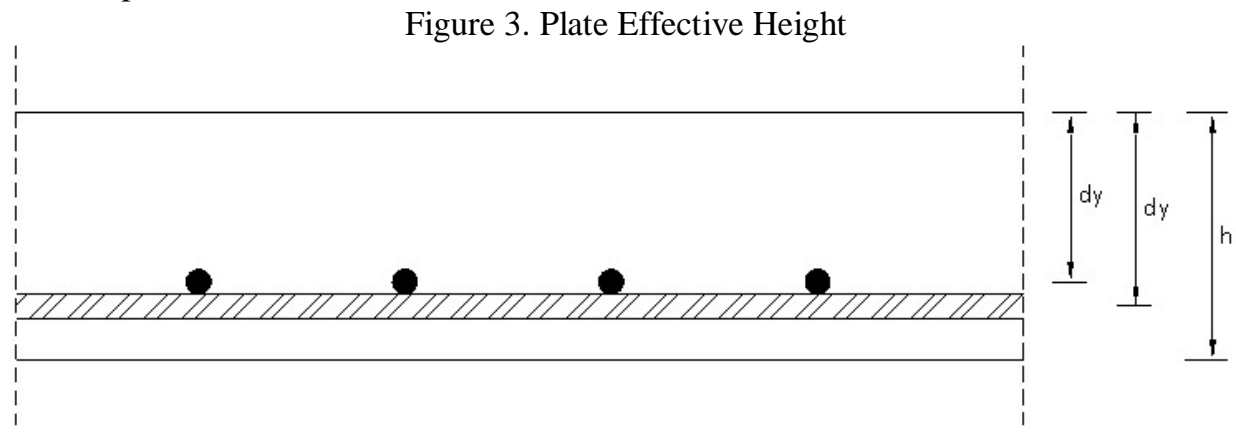

\subsection{Beam Planning}

In the pre-designed beam height according to (SNI 03-2847-2002, n.d.)is the function and span and quality of the concrete used. In general, the pre-designed beam height is $\mathrm{L} / 10-\mathrm{L} / 15$, and the beam width is taken as $1 / 2 \mathrm{H}$ $-2 / 3 \mathrm{H}$ where $\mathrm{H}$ is the beam height. Calculation of internal forces on the beam using Avizan \& Koespiadi, (2020)software. From the results of the output of these internal forces, they are then used to calculate the reinforcement requirements.

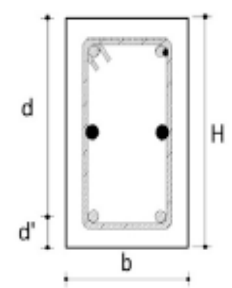

$d^{\prime}=p+\emptyset$ stirrup $+1 / 2 \varnothing$ main reinforcement

$d=h-\left(p+\emptyset\right.$ stirrup $+1 \frac{1}{2} \varnothing$ main reinforcement $)$

Figure .4. Plate Effective Height

\subsection{Coloumn Planning}

Column elements receive bending loads and axial loads, according to (SNI 03-2847-2002, n.d.)article 11.3.2.2. for planning columns that receive bending and axial loads, the material reduction coefficient is set at 0.65 , while the distribution of reinforcement in columns (rectangular cross-section) can be done by installing symmetrical reinforcement on two sides of the column (two faces) or installing reinforcement on four sides of the column (four faces).

In planning this apartment building, column planning is used using reinforcement on four sides of the column cross section (four faces). Calculation of internal forces on the column using the Avizan \& Koespiadi, (2020)program.

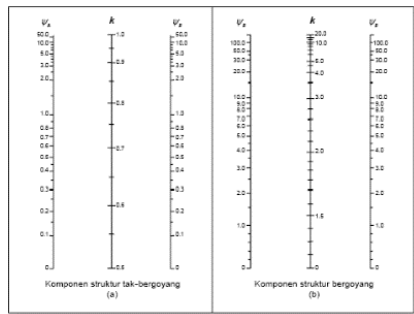

Figure 5. Effective Length Factor (k)

This work is licensed under a Creative Commons Attribution-ShareAlike 4.0 International License. 


\section{Result And Analysis}

The results and discussion contain the following descriptions:

\subsection{Structure Data}

The building characteristics data are as follows:

a) 38-storey apartment building with a total height of $118.5 \mathrm{~m}$

b) The height of the basement floor and 1st floor is $4.5 \mathrm{~m}$ which is used for parking, the height of the 2nd and 3rd floors is $3 \mathrm{~m}$ which is used as a commercial area and the height of the 3rd to 37th floors is $3 \mathrm{~m}$ which is functioned as a residence.

c) The main structure is planned to use the Special Moment Bearing Frame System with the construction of Shearwall, Columns and Beams using reinforced concrete structures, floor plates and roof plates using reinforced concrete slabs.

Figure 6. 3D Building Structure System

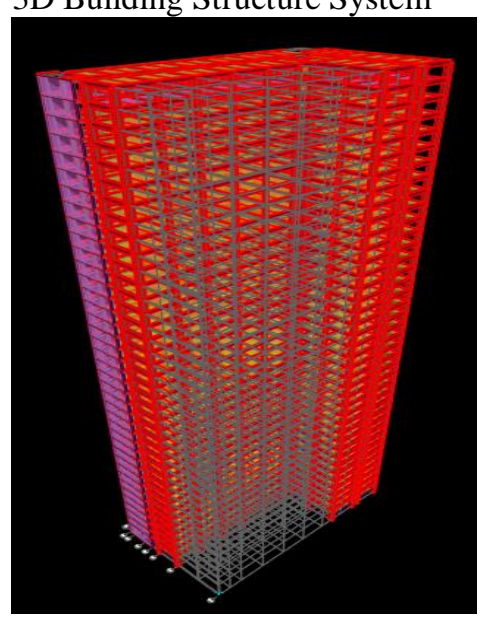

Figure 7. Layout Lt. $1-3$

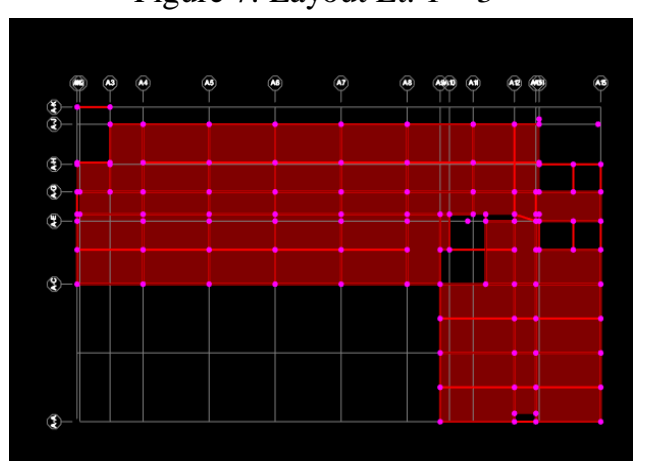

Figure 9. Layout Lt. 5 - 38

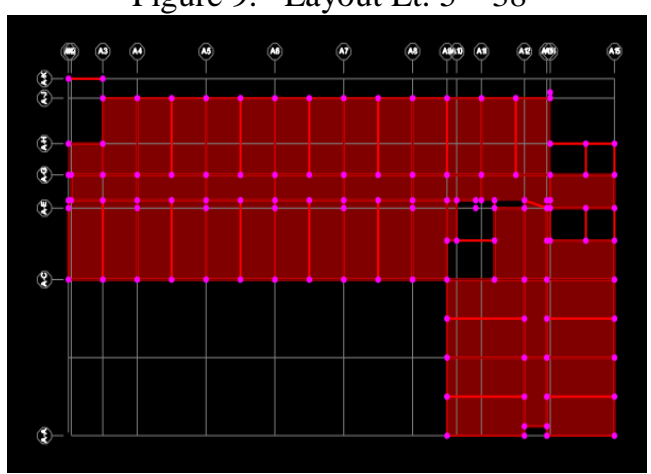

Figure 8. Layout Lt. 4

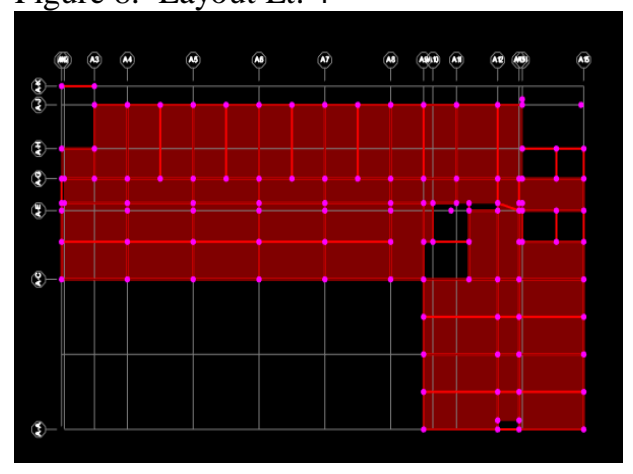

Figure. 10. Layout Lt. Roof

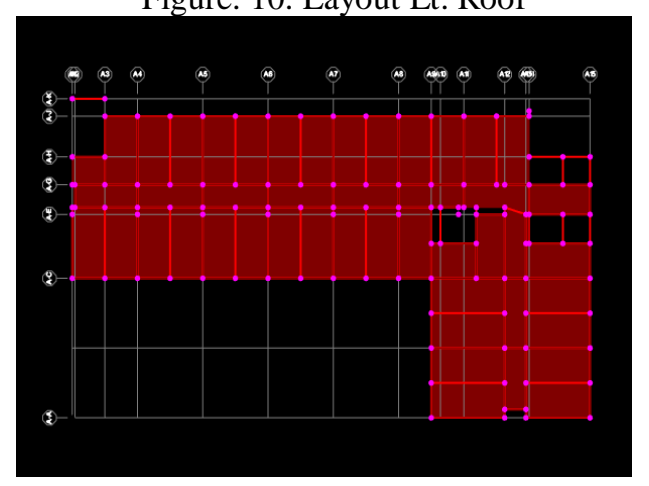

This work is licensed under a Creative Commons Attribution-ShareAlike 4.0 International License. 
Table 1. Columns and ShearWall

\begin{tabular}{|c|c|c|c|c|c|c|c|c|c|}
\hline \multirow{2}{*}{ Floor } & & \multicolumn{7}{|c|}{ TYPE COLOUMN (mm) } & \multirow{2}{*}{$\begin{array}{c}\mathrm{F}^{\prime} \mathrm{C} \\
\text { (Mpa) }\end{array}$} \\
\hline & C5 & C8 & $\mathrm{Cg}$ & $\mathrm{C} 10$ & C11 & C11A & C11B & C12 & \\
\hline$-1 \mathrm{~F}$ & $1200 \times 700$ & $1000 \times 700$ & $1200 \times 600$ & $1200 \times 600$ & $1200 \times 600$ & $1000 \times 900$ & $1100 \times 800$ & $1300 \times 600$ & 50 \\
\hline $1 F-2 F$ & $1200 \times 7001$ & $1000 \times 700$ & $1200 \times 600$ & $1200 \times 600$ & $1200 \times 600$ & $1000 \times 900$ & $1100 \times 800$ & $1300 \times 600$ & 50 \\
\hline $3 F-J C / 4 F$ & $1200 \times 600$ & $1000 \times 700$ & $1200 \times 600$ & $1200 \times 600$ & $1200 \times 600$ & $1000 \times 800$ & $1100 \times 800$ & $1300 \times 600$ & 50 \\
\hline $5 F$ & $1200 \times 6001$ & $1000 \times 700$ & $1200 \times 600$ & $1200 \times 600$ & $1200 \times 600$ & $1000 \times 700$ & $1100 \times 700$ & $1300 \times 600$ & 50 \\
\hline $6 F-7 F$ & $1200 \times 600$ & $1000 \times 600$ & $1100 \times 600$ & $1200 \times 600$ & $1100 \times 600$ & $1000 \times 700$ & $1100 \times 700$ & $1200 \times 500$ & 50 \\
\hline $8 F-11 F$ & $1200 \times 600$ & $1000 \times 600$ & $1100 \times 600$ & $1200 \times 600$ & $1100 \times 600$ & $1000 \times 700$ & $1100 \times 600$ & $1200 \times 500$ & 45 \\
\hline $12 F-13 F$ & $1200 \times 500$ & $1000 \times 500$ & $1000 \times 600$ & $1100 \times 600$ & $1000 \times 600$ & $1000 \times 600$ & $1100 \times 600$ & $1200 x$ & 45 \\
\hline $14 \mathrm{~F}-1$ & $1200 \times 500$ & $1000 \times 500$ & $1000 \times 600$ & $1100 \times 600$ & $1000 \times 500$ & $1000 \times 600$ & $1100 \times 600$ & $1200 x$ & 40 \\
\hline $19 \mathrm{~F}$ & $1200 \times 5001$ & $1000 \times 400$ & $900 \times 600$ & $1100 \times 600$ & $1000 \times 500$ & $1000 \times 500$ & & & 40 \\
\hline $20 \mathrm{~F}-23 \mathrm{~F}$ & $1200 \times 5001$ & $1000 \times 400$ & $900 \times 600$ & $1100 \times 600$ & $1000 \times 400$ & $1000 \times 500$ & $1100 \times 500$ & $1200 x$ & 35 \\
\hline $24 \mathrm{~F}-29 \mathrm{~F}$ & $1100 \times 5001$ & $1000 \times 400$ & $900 \times 500$ & $1000 \times 600$ & $1000 \times 400$ & $1000 \times 400$ & $1000 \times 500$ & & 35 \\
\hline $30 \mathrm{~F}-38 \mathrm{~F}$ & & & $900 \times 400$ & $1000 \times 600$ & $900 \times 400$ & & & & 35 \\
\hline & & & & TYPE COLO & UUMN (mm) & & & & $\mathrm{F}^{\prime} \mathrm{C}$ \\
\hline Hloor & $\mathrm{C} 1$ & C1A & $\mathrm{C} 1 \mathrm{~B}$ & $\begin{array}{ll}\mathrm{C} 2 \\
\end{array}$ & \begin{tabular}{l|l}
$\mathrm{C}$ \\
$\mathrm{A}$
\end{tabular} & C3 & C4 & C4A & (Mpa) \\
\hline$-1 F$ & $1200 \times 600$ & $1300 \times 600$ & $1200 \times 600$ & \begin{tabular}{|l|l|}
0 & $1200 \times 600$ \\
\end{tabular} & $1200 \times 500$ & $1300 \times 600$ & $1200 \times 700$ & $1200 x$ & 50 \\
\hline $1 F-2 F$ & $1200 \times 600$ & $1300 \times 600$ & $1200 \times 600$ & $01200 \times 600$ & $1100 \times 500$ & $1200 \times 600$ & $1200 \times 700$ & $1200 \times 600$ & 50 \\
\hline $3 F-J C / 4 F$ & $1200 \times 600$ & $1200 \times 600$ & $1100 \times 600$ & $0 \quad 1100 \times 600$ & $1100 \times 500$ & $1200 x$ & $1200 \times 600$ & & 50 \\
\hline $5 F$ & $1100 \times 600$ & $1200 \times 600$ & $1100 \times 600$ & $0 \quad 1100 \times 600$ & $1100 \times 500$ & $1200 \times 600$ & $1200 \times 600$ & & 50 \\
\hline $6 F-7 F$ & $1100 \times 600$ & $1200 \times 600$ & $1100 \times 600$ & $01100 \times 600$ & $1100 \times 500$ & $1200 \times 500$ & $1100 \times 600$ & 1100 & 45 \\
\hline $8 \mathrm{~F}-11 \mathrm{~F}$ & $1000 \times 600$ & $1200 \times 600$ & $1100 \times 600$ & $01100 \times 600$ & $1100 \times 500$ & $1200 \times 500$ & $1100 \times 600$ & $1100 x$ & 45 \\
\hline $12 \mathrm{~F}-13 \mathrm{~F}$ & $1000 \times 600$ & $1100 \times 600$ & $1000 \times 600$ & \begin{tabular}{l|l|}
0 & $1000 \times 600$ \\
\end{tabular} & $1000 \times 500$ & $1100 \times 500$ & $1000 \times 600$ & $1000 \times 600$ & 40 \\
\hline $14 \mathrm{~F}-18 \mathrm{~F}$ & $1000 \times 600$ & $1100 \times 600$ & $1000 \times 600$ & $\begin{array}{lll}0 & 1000 \times 500 \\
\end{array}$ & $1000 \times 500$ & $1100 \times 500$ & $1000 \times 600$ & & 40 \\
\hline $19 F$ & $1000 \times 500$ & $1100 \times 500$ & $1000 \times 500$ & $\begin{array}{ll}0 & 1000 \times 500 \\
\end{array}$ & $1000 \times 500$ & $1100 x$ & $1000 \times 500$ & 1000 & 35 \\
\hline $20 \mathrm{~F}-23 \mathrm{~F}$ & $1000 \times 400$ & $1000 \times 500$ & $1000 \times 500$ & $01000 \times 500$ & $1000 \times 500$ & $1000 \times 500$ & $1000 \times 500$ & $1000 x$ & 35 \\
\hline $24 \mathrm{~F}-29 \mathrm{~F}$ & $1000 \times 400$ & $1000 \times 500$ & $1000 \times 400$ & $01000 \times 400$ & $1000 \times 400$ & $1000 \times 400$ & $1000 \times 400$ & $1000 x$ & 35 \\
\hline $30 \mathrm{~F}-38 \mathrm{~F}$ & $900 \times 400$ & $1000 \times 400$ & $900 \times 400$ & $900 \times 400$ & $900 \times 400$ & $1000 \times 400$ & $900 \times 400$ & $900 \times 400$ & 35 \\
\hline & & & & & 1 & & & & \\
\hline & Floor & SW & & SW2 & SW3 & & W4 & & \\
\hline & & $\mathrm{P}=75$ & & $=1600$ & $P=6400$ & 0 & 4250 & & \\
\hline & $-11 F$ & & 550 & 550 & & & & 50 & \\
\hline & $-23 F$ & & 450 & 450 & 500 & & 400 & 45 & \\
\hline & $\mathrm{F}-\mathrm{ROOF}$ & & 350 & 350 & & & & 40 & \\
\hline
\end{tabular}

This work is licensed under a Creative Commons Attribution-ShareAlike 4.0 International License. 
Table 2. Beam

\begin{tabular}{|c|c|c|c|c|}
\hline Lantai & Tipe & Balok & Properti & $\mathrm{F}^{\prime} \mathrm{C}(\mathrm{Mpa})$ \\
\hline Balok Lt. 1 & B412 & Beton & $400 \times 1200$ & 40 \\
\hline Balok Lt. 1 & B412A-2 & Beton & $400 \times 1130$ & 40 \\
\hline Balok Lt. 1 & B311A & Beton & $300 \times 780$ & 40 \\
\hline Balok Lt. 1 & B73A & Beton & $700 \times 350$ & 40 \\
\hline Balok Lt. 1 & B66 & Beton & $600 \times 600$ & 40 \\
\hline Balok Lt. 1 & B66A & Beton & $600 \times 650$ & 40 \\
\hline Balok Lt. 1 - 4 & B2A4 & Beton & $250 \times 400$ & 40 \\
\hline Balok Lt. 1 - 4 & B2A5 & Beton & $250 \times 500$ & 40 \\
\hline Balok Lt. 1 - 4 & B4A7A & Beton & $450 \times 750$ & 40 \\
\hline Balok Lt. 1 - 4 & B76A & Beton & $700 \times 650$ & 40 \\
\hline Balok Lt. 1 - 4 & B45 & Beton & $400 \times 500$ & 40 \\
\hline Balok Lt. 1 - 4 & B47 & Beton & $400 \times 700$ & 40 \\
\hline Balok Lt. 1 - 4 & B4A10 & Beton & $450 \times 1000$ & 40 \\
\hline Balok Lt. 5 - 24 & B2A5A & Beton & $250 \times 550$ & 35 \\
\hline Balok Lt. 5 - 24 & B2A6A & Beton & $250 \times 650$ & 35 \\
\hline Balok Lt. 5 - 24 & B3A5A & Beton & $350 \times 550$ & 35 \\
\hline Balok Lt. 5 - 24 & B3A5A 1 & Beton & $350 \times 560$ & 35 \\
\hline Balok Lt. 5 - 24 & B2A7A3 & Beton & $250 \times 780$ & 35 \\
\hline Balok Lt. 5 - 24 & B37A3 & Beton & $300 \times 780$ & 35 \\
\hline Balok Lt. 5 - 24 & B57 & Beton & $500 \times 700$ & 35 \\
\hline Balok Lt. 5 - 24 & B59 & Beton & $500 \times 900$ & 35 \\
\hline Balok Lt. 5 - 24 & B410A3 & Beton & $400 \times 1080$ & 35 \\
\hline Balok Lt. 5 - 24 & B612 & Beton & $600 \times 1200$ & 35 \\
\hline Balok Lt. 5 - 24 & B69 & Beton & $600 \times 900$ & 35 \\
\hline Balok Lt. 5 - 24 & B46 & Beton & $400 \times 600$ & 35 \\
\hline Balok Lt. 5 - 24 & B 103A & Beton & $1000 \times 350$ & 35 \\
\hline Balok Lt. 5 - 24 & B93A & Beton & $900 \times 350$ & 35 \\
\hline Balok Lt. $25-38$ & B59 & Beton & $500 \times 900$ & 35 \\
\hline Balok Lt. 25 - 38 & B46A & Beton & $300 \times 500$ & 35 \\
\hline Balok Lt. $25-38$ & B55A & Beton & $300 \times 500$ & 35 \\
\hline Balok Lt. 25 - 38 & B56A & Beton & $300 \times 500$ & 35 \\
\hline Balok Lt. $25-38$ & B47A & Beton & $300 \times 500$ & 35 \\
\hline Balok Lt. 25 - 38 & B45A & Beton & $300 \times 500$ & 35 \\
\hline Balok Lt. 25 - 38 & B35A & Beton & $300 \times 500$ & 35 \\
\hline Balok Lt. 25 - 38 & B83A & Beton & $800 \times 350$ & 35 \\
\hline Balok Lt. 25 - 38 & B45A & Beton & $300 \times 500$ & 35 \\
\hline Balok Lt. 25 - 38 & B35A & Beton & $300 \times 500$ & 35 \\
\hline Balok Lt. $25-38$ & B2A6A & Beton & $300 \times 500$ & 35 \\
\hline Balok Lt. $25-38$ & B34A 1 & Beton & $300 \times 500$ & 35 \\
\hline Balok Lt. 5 - 38 & B2A4 & Beton & $300 \times 500$ & 35 \\
\hline Balok Lt. 5 - 38 & B23 & Beton & $300 \times 500$ & 35 \\
\hline Balok Lt. 5 - 38 & B24 & Beton & $300 \times 500$ & 35 \\
\hline Balok Lt. 5 - 38 & B24" & Beton & $300 \times 500$ & 35 \\
\hline Balok Lt. 5 - 38 & B25 & Beton & $300 \times 500$ & 35 \\
\hline Balok Lt. 5 - 38 & B2A3A & Beton & $300 \times 500$ & 35 \\
\hline Balok Lt. 5 - 38 & B4A6 & Beton & $300 \times 500$ & 35 \\
\hline
\end{tabular}

Table 3. Thickness of Concrete Slab

\begin{tabular}{clc}
\hline \multicolumn{2}{c}{ floor } & Tebal $(\mathrm{mm})$ \\
\hline Graund flor & concrete & 150 \\
Floor 1 & concrete & 150 \\
Floor 2 & concrete & 130 \\
Floor 3-4 & concrete & 120 \\
Floor 5-38 & concrete & 120 \\
Ro of top & concrete & 150 \\
\hline
\end{tabular}

Table 4. Quality Material

\begin{tabular}{ccc}
\hline Structure element & Cobcrete grade & Component character \\
\hline Raft foundation & FC35 & 35 \\
Coloumn and shearwall & FC /3540/45/50 & $35 / 40 / 45 / 50$ \\
Girder / beam & FC 35 & 35 \\
slab & FC 35 & 35 \\
\hline
\end{tabular}

This work is licensed under a Creative Commons Attribution-ShareAlike 4.0 International License. 
E-ISSN: 2685-3272 | P-ISSN 1412-0860

Table 5. Structure element

\begin{tabular}{ccc}
\hline Structure element & Cobcrete grade & Component character \\
\hline Raft foundation & FC35 & 35 \\
Coloumn and shearwall & FC /3540/45/50 & $35 / 40 / 45 / 50$ \\
Girder / beam & FC 35 & 35 \\
slab & FC 35 & 35 \\
\hline
\end{tabular}

\subsection{Loading}

\section{Static Load}

Static analysis in principle is to replace earthquake loads with seismic forces static equivalent which aims to simplify and simplify calculations. This method is also called the Equivalent Lateral Force Method, which assumes the magnitude of the earthquake force based on the product of a constant/mass of the element.

Table 6. Dead Load of Construction Materials and Building Components

\begin{tabular}{clc}
\hline No & \multicolumn{1}{c}{ Material konstruksi } & $\left(\mathrm{Kg} / \mathrm{m}^{3}\right)$ \\
\hline 1 & Steel & 7850 \\
2 & Conciente & 2200 \\
3 & Reinforced ConCrete & 2500 \\
4 & Wood ( Everage Value of different types of wood) & 1000 \\
5 & Red Bricks & 1700 \\
6 & Pair Of split stones, cobblestones, mountain stones & 2200 \\
7 & Sand (air dry to moist) & $1600-1700$ \\
8 & Sand (saturated with water ) & 1800 \\
9 & Soll,logam and still (dry - wet ) & $1700-2000$ \\
10 & split & 1450 \\
\hline
\end{tabular}

Table 7. Self Weight of Building Components

\begin{tabular}{|c|c|c|}
\hline $\mathrm{NO}$ & Komponen gedung & $\begin{array}{c}\text { Beban mati } \\
(\mathrm{kg} / \mathrm{m} 2)\end{array}$ \\
\hline \multirow[t]{3}{*}{1} & Stir ( per cm think ) & \\
\hline & -From cement & 21 \\
\hline & - Of lime, red cement or tras & 17 \\
\hline \multirow[t]{3}{*}{2} & Ceiling (including ribs, whithout hangers) & \\
\hline & -Asbestos cement /plasterboard ( $\max 4 \mathrm{~mm}$ thick ) & 11 \\
\hline & Glass $(3-5 \mathrm{~mm}$ Thick $)$ & 10 \\
\hline \multirow[t]{3}{*}{3} & Red mas onry wall & \\
\hline & -one stone & 450 \\
\hline & -half a stone & 250 \\
\hline \multirow[t]{5}{*}{4} & Brick ma sonry wall & \\
\hline & -perforated $(20 \mathrm{~cm}$ thick $)$ & 200 \\
\hline & -perforated (10 cm thick ) & 120 \\
\hline & -no holes (15 cm thick ) & 300 \\
\hline & no holes $(10 \mathrm{~cm}$ thick $)$ & 200 \\
\hline 5 & Wave asbestos cement ( $5 \mathrm{~mm}$ think $) 11$ & 11 \\
\hline 6 & Simple wooden floor, no celling & 40 \\
\hline 7 & Wooden ceiling hanger ( $\max 5 \mathrm{~m}$ span) & 7 \\
\hline 8 & Cemment/concrete floor coverings (per $\mathrm{cm}$ thick) & 24 \\
\hline
\end{tabular}

Table 8. Live Load On Structure

\begin{tabular}{cc}
\hline Live loads on Floors of large building & Burden \\
\hline Floor $250 \mathrm{~kg} \mathrm{~m} \mathrm{~m}^{2}$ & $250 \mathrm{~kg} / \mathrm{m}^{2}$ \\
Stairs and beban $300 \mathrm{~kg} / \mathrm{m}^{2}$ & $300 \mathrm{~kg} / \mathrm{m}^{2}$ \\
Roof plate $100 \mathrm{~kg} / \mathrm{m}^{2}$ & $100 \mathrm{~kg} / \mathrm{m}^{2}$ \\
Floor tool and marchine room $400 \mathrm{~kg} / \mathrm{m}^{2}$ & $400 \mathrm{~kg} / \mathrm{m}^{2}$ \\
\hline
\end{tabular}

2. Dynamic Load

This work is licensed under a Creative Commons Attribution-ShareAlike 4.0 International License. 
Table 9. Definition of Soil Type (SNI 1726-2002)

\begin{tabular}{|c|c|c|c|}
\hline Type of soil & $\begin{array}{l}\text { Average shear wave } \\
\text { velocity }(\mathrm{vs})(\mathrm{m} / \mathrm{s})\end{array}$ & $\begin{array}{l}\text { The average standard } \\
\text { penetration test result } \\
\text { value }(\mathrm{N})\end{array}$ & $\begin{array}{l}\text { Average shear streght } \mathrm{u} \\
\qquad(\mathrm{kPa})\end{array}$ \\
\hline Hard soil & Vs 350 & $\mathrm{~N} \geq 50$ & $\mathrm{su} \geq 100$ \\
\hline Medium graund & $175 \leq \mathrm{vs}<350$ & $15 \leq \mathrm{N}<50$ & $50 \leq \mathrm{su}<100$ \\
\hline Soft soil & $V_{s}<175$ & $<15$ & \\
\hline Soft soi & $\mathrm{Vs}<175$ & $<15$ & \\
\hline Special and & \multicolumn{3}{|c|}{$\begin{array}{c}\text { Or }<\text { all types of soft clay with a total thickness of more than } 3 \text { meter wiyh } \\
\text { PI }>20 . w n 40 \% \text { and } u<25 \mathrm{Kpa} \\
\text { A special evalution is required at each location }\end{array}$} \\
\hline
\end{tabular}

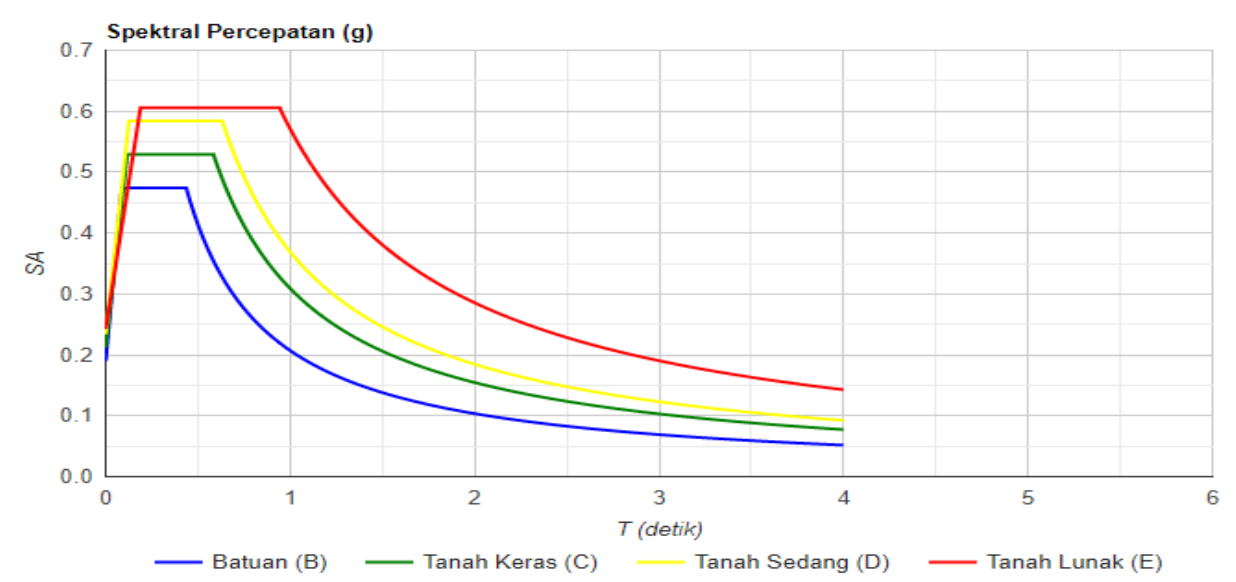

Figure 11 .Response Spectrum for the AlamSutera Areas 
E-ISSN: 2685-3272 | P-ISSN 1412-0860

Table 10. Spectral Results Table for the AlamSutera Areas

\begin{tabular}{|c|c|c|c|c|c|c|c|c|c|c|c|}
\hline \multirow[t]{3}{*}{ Rock } & Variable & Point & \multirow{3}{*}{$\begin{array}{l}\text { Hard } \\
\text { Soil }\end{array}$} & Variable & Point & \multirow{3}{*}{\begin{tabular}{|l} 
Medium \\
Ground \\
/ Soil \\
\end{tabular}} & Variable & Point & \multirow{3}{*}{\begin{tabular}{|l|} 
Soft \\
Soil
\end{tabular}} & Variable & Point \\
\hline & PGA (g) & 0,373 & & PGA (g) & 0,373 & & PGA (g) & 0,373 & & PGA (g) & 0,373 \\
\hline & $\mathrm{S}_{\mathrm{S}}(\mathrm{g})$ & 0,712 & & $\mathrm{~S}_{\mathrm{S}}(\mathrm{g})$ & 0,712 & & $\mathrm{~S}_{\mathrm{S}}(\mathrm{g})$ & 0,712 & & $\mathrm{~S}_{\mathrm{S}}(\mathrm{g})$ & 0,712 \\
\hline & $\mathrm{S}_{1}(\mathrm{~g})$ & 0,311 & & $\mathrm{~S}_{1}(\mathrm{~g})$ & 0,311 & & $\mathrm{~S}_{1}(\mathrm{~g})$ & 0,311 & & $\mathrm{~S}_{1}(\mathrm{~g})$ & 0,311 \\
\hline & $\mathrm{C}_{\mathrm{RS}}$ & 0,996 & & $\mathrm{C}_{\mathrm{RS}}$ & 0,996 & & $\mathrm{C}_{\mathrm{RS}}$ & 0,996 & & $\mathrm{C}_{\mathrm{RS}}$ & 0,996 \\
\hline & $\mathrm{C}_{\mathrm{R} 1}$ & 0,936 & & $\mathrm{C}_{\mathrm{R} 1}$ & 0,936 & & $\mathrm{C}_{\mathrm{R} 1}$ & 0,936 & & $\mathrm{C}_{\mathrm{R} 1}$ & 0,936 \\
\hline & $\mathrm{F}_{\mathrm{PGA}}$ & 1 & & $\mathrm{~F}_{\mathrm{PGA}}$ & 1,027 & & $\mathrm{~F}_{\mathrm{PGA}}$ & 1,127 & & $\mathrm{~F}_{\mathrm{PGA}}$ & 0,98 \\
\hline & $\mathrm{F}_{\mathrm{A}}$ & 1 & & $\mathrm{~F}_{\mathrm{A}}$ & 1,115 & & $\mathrm{~F}_{\mathrm{A}}$ & 1,231 & & $\mathrm{~F}_{\mathrm{A}}$ & 1,277 \\
\hline & $\mathrm{F}_{\mathrm{V}}$ & 1 & & $\mathrm{~F}_{\mathrm{V}}$ & 1,489 & & $\mathrm{~F}_{\mathrm{V}}$ & 1,778 & & $\mathrm{~F}_{\mathrm{V}}$ & 2,756 \\
\hline & PSA (g) & 0,373 & & PSA (g) & 0,383 & & PSA (g) & 0,421 & & PSA (g) & 0,366 \\
\hline & $\mathrm{S}_{\mathrm{MS}}(\mathrm{g})$ & 0,712 & & $\mathrm{~S}_{\mathrm{MS}}(\mathrm{g})$ & 0,794 & & $\mathrm{~S}_{\mathrm{MS}}(\mathrm{g})$ & 0,876 & & $S_{M S}(g)$ & 0,909 \\
\hline & $\mathrm{S}_{\mathrm{M} 1}(\mathrm{~g})$ & 0,311 & & $\mathrm{~S}_{\mathrm{M} 1}(\mathrm{~g})$ & 0,463 & & $\mathrm{~S}_{\mathrm{M} 1}(\mathrm{~g})$ & 0,553 & & $\mathrm{~S}_{\mathrm{M} 1}(\mathrm{~g})$ & 0,857 \\
\hline & $\mathrm{S}_{\mathrm{DS}}(\mathrm{g})$ & 0,474 & & $\mathrm{~S}_{\mathrm{DS}}(\mathrm{g})$ & 0,529 & & $\mathrm{~S}_{\mathrm{DS}}(\mathrm{g})$ & 0,584 & & $\mathrm{~S}_{\mathrm{DS}}(\mathrm{g})$ & 0,606 \\
\hline & $\mathrm{S}_{\mathrm{D} 1}(\mathrm{~g})$ & 0,207 & & $\mathrm{~S}_{\mathrm{D} 1}(\mathrm{~g})$ & 0,309 & & $\mathrm{~S}_{\mathrm{D} 1}(\mathrm{~g})$ & 0,369 & & $\mathrm{~S}_{\mathrm{D} 1}(\mathrm{~g})$ & 0,571 \\
\hline & $\mathrm{T}_{0}$ (detik) & 0,087 & & $\mathrm{~T}_{0}$ (detik) & 0,117 & & $\mathrm{~T}_{0}$ (detik) & 0,126 & & $\mathrm{~T}_{0}$ (detik) & 0,189 \\
\hline & $\mathrm{T}_{\mathrm{S}}$ (detik) & 0,437 & & $\mathrm{~T}_{\mathrm{S}}$ (detik) & 0,583 & & $\mathrm{~T}_{\mathrm{S}}($ detik) & 0,631 & & $\mathrm{~T}_{\mathrm{S}}($ detik) & 0,944 \\
\hline
\end{tabular}

Table 11. Priority Factors For Various Categories Of Buildings

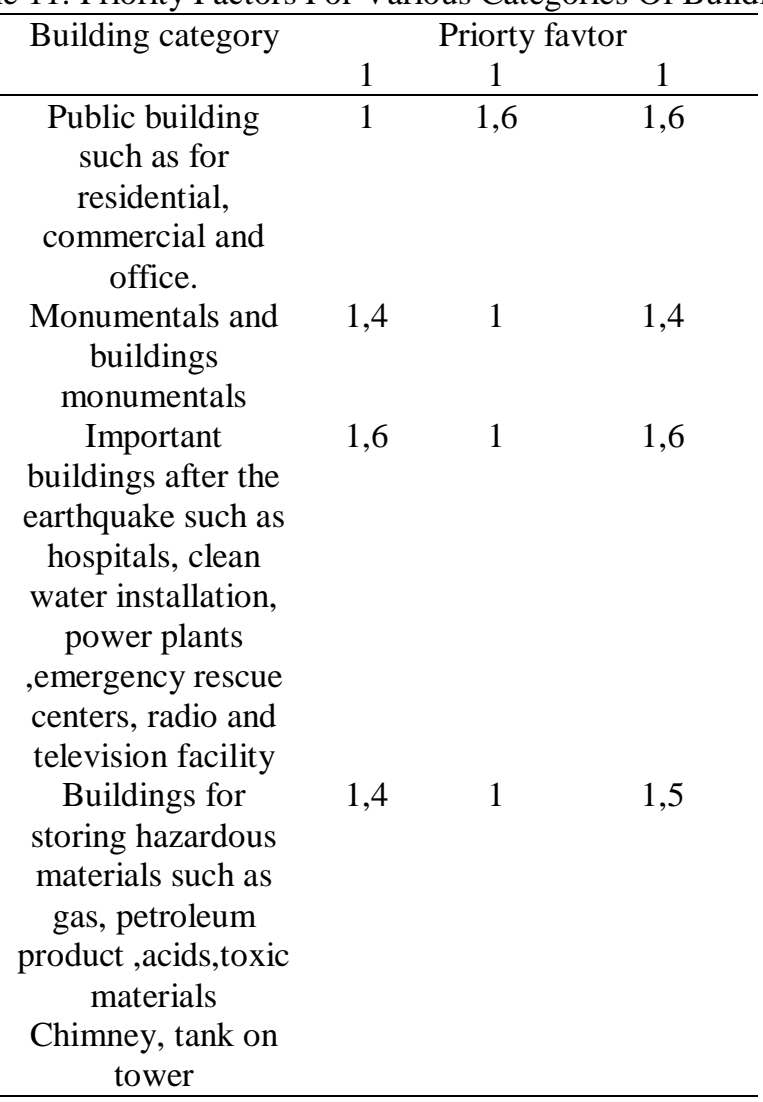




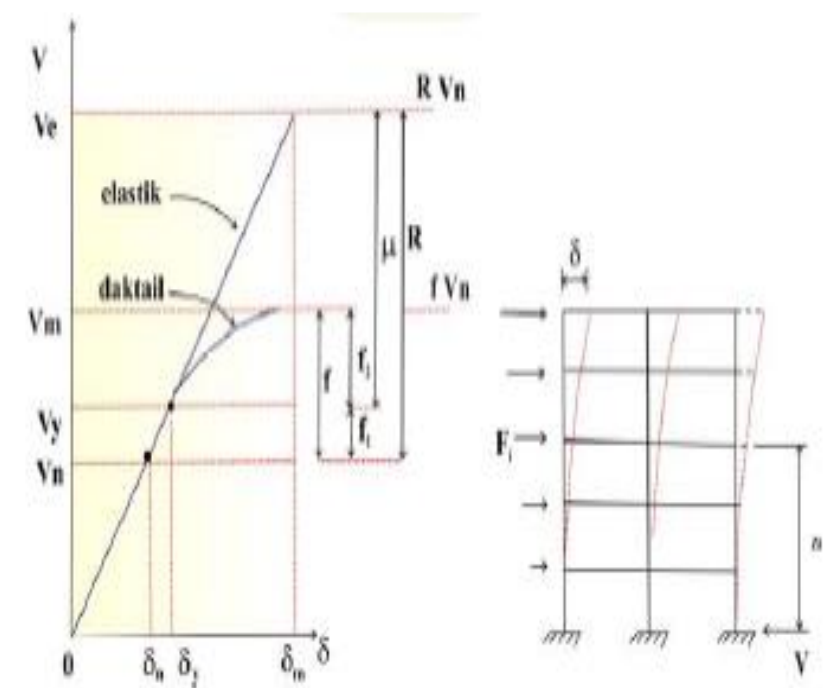

Figure 12. Load Diagram (V) - Deviation $(\delta)$ of the Building Structure

Table 12. Peak acceleration of bedrock and peak ground acceleration for each Indonesian Earthquake Region

\begin{tabular}{cccccc}
\hline $\begin{array}{c}\text { eartgua ke } \\
\text { region }\end{array}$ & $\begin{array}{c}\text { Peak } \\
\text { acceleration } \\
\text { of bedrock }\end{array}$ & Hard soil & Medium & Soft soil & Special land \\
('g') & 0,03 & 0,04 & 0,05 & 0,08 & A special \\
\hline 1 & 0,1 & 0,12 & 0,15 & 0,2 & evaluation \\
2 & 0,15 & 0,18 & 0,23 & 0,3 & is required \\
3 & 0,2 & 0,24 & 0,28 & 0,34 & at each \\
4 & 0,25 & 0,28 & 0,32 & 0,36 & location \\
5 & 0,3 & 0,33 & 0,36 & 0,38 & \\
6 & & & & & \\
\hline
\end{tabular}

Table 13. Coefficient of Vibration Time of Earthquake Area Structure

\begin{tabular}{cc}
\hline Earthquake region & $\begin{array}{c}\text { Limiting coefficient } \\
\text { ('g') }\end{array}$ \\
\hline 1 & 0,2 \\
2 & 0,19 \\
3 & 0,18 \\
4 & 0,17 \\
5 & 0,16 \\
6 & 0,15 \\
\hline
\end{tabular}

Table 14. Estimated Amount of Ksv Price for Several Types of Land

\begin{tabular}{cc}
\hline Type of soil & $\begin{array}{c}\text { Range price ksw } \\
(\mathrm{kN} / \mathrm{m} 3)\end{array}$ \\
\hline sand & $4500-15000$ \\
Loose sand & $9000-75000$ \\
Medium sand & $60000-120000$ \\
Dense sand & $30000-75000$ \\
Clayey sand & $22500-45000$ \\
Silty sand & \\
Clay & $11250-22500$ \\
Qu $<4 \mathrm{kPa}$ & $22500-45000$ \\
$4 \mathrm{kPa}<\mathrm{qu}<8 \mathrm{kPa}$ & $>45000$ \\
$8 \mathrm{kPa}<\mathrm{qu}$ & \\
\hline
\end{tabular}

This work is licensed under a Creative Commons Attribution-ShareAlike 4.0 International License. 
Table 15. Maximum Permit Deflection

\begin{tabular}{|c|c|c|}
\hline Structural Component Type & Calculated Deflection & Limit Deflection \\
\hline $\begin{array}{l}\text { Flat roofs that do not support } \\
\text { or are not attached to non- } \\
\text { structural components that } \\
\text { may be damaged by large } \\
\text { deflections }\end{array}$ & $\begin{array}{l}\text { Instantaneous deflection } \\
\text { due to live load (LL) }\end{array}$ & $\frac{l^{a}}{180}$ \\
\hline $\begin{array}{l}\text { Floors that do not support or } \\
\text { are not attached to non- } \\
\text { structural components that are } \\
\text { likely to be damaged by large } \\
\text { deflections }\end{array}$ & $\begin{array}{l}\text { Instantaneous deflection } \\
\text { due to live load (LL) }\end{array}$ & $\frac{!}{360}$ \\
\hline $\begin{array}{l}\text { Roof or floor construction that } \\
\text { supports or is attached to non- } \\
\text { structural components that are } \\
\text { likely to be damaged by large } \\
\text { deflections }\end{array}$ & \multirow{2}{*}{$\begin{array}{l}\text { Part of the total } \\
\text { deflection that occurs } \\
\text { after the installation of } \\
\text { non-structural } \\
\text { components (sum of the } \\
\text { long-term deflection, due } \\
\text { to all fixed loads acting, } \\
\text { and the instantaneous } \\
\text { deflection, due to the } \\
\text { addition of live loads) }\end{array}$} & $\frac{l^{b}}{480}$ \\
\hline $\begin{array}{l}\text { Roof or floor construction that } \\
\text { supports or is attached to non- } \\
\text { structural components that are } \\
\text { unlikely to be damaged by } \\
\text { large deflections. }\end{array}$ & & \\
\hline \multicolumn{3}{|c|}{$\begin{array}{l}\text { These limits are not intended to prevent possible waterlogging. The } \\
\text { possibility of inundation should be checked by calculating the deflection, } \\
\text { including the additional deflection due to the inundation, and taking into } \\
\text { account the long-term effects of the always-acting load, deflection } \\
\text { resistance, construction tolerances and the reliability of the drainage system. }\end{array}$} \\
\hline \multicolumn{3}{|c|}{$\begin{array}{l}\text { b The deflection limit may be exceeded when steps have been taken to } \\
\text { prevent damage to the supported or joined components. }\end{array}$} \\
\hline \multicolumn{3}{|c|}{$\begin{array}{l}\text { Long-term deflection shall be calculated according to the provisions of } \\
11.5(2(5)) \text { or } 11.5(4(2)) \text {, but may be reduced by the value of the deflection } \\
\text { occurring before the addition of non-structural components. The value of } \\
\text { this deflection must be determined on the basis of acceptable technical data } \\
\text { regarding the characteristics of the relationship between time and deflection } \\
\text { of the structural member which is similar to that of the member under } \\
\text { review }\end{array}$} \\
\hline \multicolumn{3}{|c|}{$\begin{array}{l}\text { d But it must not be greater than the tolerance provided for non-structural } \\
\text { components. This limit may be exceeded if there is a counter deflection } \\
\text { provided such that the total deflection minus the counter deflection does not } \\
\text { exceed the existing deflection limit. }\end{array}$} \\
\hline
\end{tabular}

3. Combination of Loading

the load combination becomes as described below:
a) 1,4 DL
b) 1,2 DL + 1,6 LL
c) $1,2 \mathrm{DL}+1 \mathrm{LL} \pm 0,3(\rho \mathrm{Qe}+0,2 \mathrm{~S} \mathrm{DS} \mathrm{DL}) \pm 1(\rho \mathrm{Qe}+0,2 \mathrm{~S} \mathrm{DS} \mathrm{DL})$
d) 1,2 DL $+1 \mathrm{LL} \pm 1(\rho \mathrm{Qe}+0,2 \mathrm{~S}$ DS DL $) \pm 0,3(\rho \mathrm{Qe}+0,2 \mathrm{~S} \mathrm{DS} \mathrm{DL})$
e) $0,9 \mathrm{DL} \pm 0,3(\rho \mathrm{Qe}+0,2 \mathrm{~S}$ DS DL $) \pm 1(\rho \mathrm{Qe}+0,2 \mathrm{~S} \mathrm{DS} \mathrm{DL})$
f) $0,9 \mathrm{DL} \pm 1(\rho \mathrm{Qe}+0,2 \mathrm{~S}$ DS DL $) \pm 0,3(\rho \mathrm{Qe}+0,2 \mathrm{~S}$ DS DL $)$

For redundancy equal to $1.3(\rho=1,3)$ and $\mathrm{S} \mathrm{DS}=0.457$, the translation results are as in Table 4.6.3.1 below 
Table 16. Combination table based on results of SAP2000 v21

\begin{tabular}{|c|c|c|c|c|c|c|c|}
\hline Combo Name & $\begin{array}{c}\text { Combo } \\
\text { Type }\end{array}$ & Case Name & $\begin{array}{l}\text { Scale } \\
\text { Factor }\end{array}$ & $\begin{array}{l}1.34 \mathrm{SDL}+1.34 \mathrm{DL}+0.5 \mathrm{~L} \\
\mathrm{~L}+0.3 \mathrm{EQX}-1 \mathrm{EQY} \\
\end{array}$ & & DX & 0.3 \\
\hline 1.4SDL + 1.4 DL & Linear Add & DEAD & 1.4 & $\begin{array}{l}\text { 1.34SDL+1.34DL+0.5L } \\
\mathrm{L}+0.3 \mathrm{EQX}-1 \mathrm{EQY}\end{array}$ & & DY & -1. \\
\hline 1.4SDL + 1.4 DL & & SUPERDEAD & 1.4 & $1.34 \mathrm{SDL}+1.34 \mathrm{DL}+0.5 \mathrm{~L}$ & & & \\
\hline $1.34 \mathrm{SDL}+1.34 \mathrm{DL}+$ & & & & $\mathrm{L}+1.0 \mathrm{EQX}+0.3 \mathrm{EQY}$ & Linear Add & DEAD & 1.34 \\
\hline $1.6 \mathrm{LL}$ & Linear Add & DEAD & 1.34 & $\begin{array}{l}\text { 1.34SDL+1.34DL+0.5L } \\
\mathrm{L}+1.0 \mathrm{EQX}+0.3 \mathrm{EQY}\end{array}$ & & SUPERDEAD & 1.34 \\
\hline $\begin{array}{l}1.34 \mathrm{SDL}+1.34 \mathrm{DL}+ \\
1.6 \mathrm{LL}\end{array}$ & & SUPERDEAD & 1.34 & \begin{tabular}{|l}
$\mathrm{L}+1.0 \mathrm{EQX}+0.3 \mathrm{EQY}$ \\
$1.34 \mathrm{SDL}+1.34 \mathrm{DL}+0.5 \mathrm{~L}$ \\
$\mathrm{~L}+1.0 \mathrm{EQX}+0.3 \mathrm{EQY}$
\end{tabular} & & Live & 0.5 \\
\hline $\begin{array}{l}1.34 \mathrm{SDL}+1.34 \mathrm{DL}+ \\
1.6 \mathrm{LL}\end{array}$ & & Live & 1.6 & $\begin{array}{l}1.34 \mathrm{SDL}+1.34 \mathrm{DL}+0.5 \mathrm{~L} \\
\mathrm{~L}+1.0 \mathrm{EQX}+0.3 \mathrm{EQY}\end{array}$ & & DX & 1. \\
\hline $\begin{array}{l}\text { 1.34SDL+1.34DL+0.5L } \\
\mathrm{L}+0.3 \mathrm{EQX}+1 \mathrm{EQY}\end{array}$ & Linear Add & DEAD & 1.34 & \begin{tabular}{|l}
$1.34 \mathrm{SDL}+1.34 \mathrm{DL}+0.5 \mathrm{~L}$ \\
$\mathrm{~L}+1.0 \mathrm{EQX}+0.3 \mathrm{EQY}$ \\
\end{tabular} & & DY & 0.3 \\
\hline 1.34SDL+1.34DL+0.5L & & |CUPEDPRA & 124 & $\begin{array}{l}\text { 1.34SDL+1.34DL+0.5L } \\
\mathrm{L}-1.0 \mathrm{EQX}-0.3 \mathrm{EQY}\end{array}$ & Linear Add & DEAD & 1.34 \\
\hline $\mathrm{L}+0.3 \mathrm{EQX}+1 \mathrm{EQY}$ & & SUPERDEAD & 1.34 & $1.34 \mathrm{SDL}+1.34 \mathrm{DL}+0.5 \mathrm{~L}$ & & SUPERDEAD & 1.34 \\
\hline $\begin{array}{l}1.34 \mathrm{SDL}+1.34 \mathrm{DL}+0.5 \mathrm{~L} \\
\mathrm{~L}+0.3 \mathrm{EQX}+1 \mathrm{EQY}\end{array}$ & & Live & 0.5 & \begin{tabular}{|l}
$\mathrm{L}-1.0 \mathrm{EQX}-0.3 \mathrm{EQY}$ \\
$1.34 \mathrm{SDL}+1.34 \mathrm{DL}+0.5 \mathrm{~L}$ \\
$\mathrm{~L}-1.0 \mathrm{EQX}-0.3 \mathrm{EQY}$
\end{tabular} & & Live & 0.5 \\
\hline $\begin{array}{l}1.34 \mathrm{SDL}+1.34 \mathrm{DL}+0.5 \mathrm{~L} \\
\mathrm{~L}+0.3 \mathrm{EQX}+1 \mathrm{EQY}\end{array}$ & & DX & 0.3 & $\begin{array}{l}1.34 \mathrm{SDL}+1.34 \mathrm{DL}+0.5 \mathrm{~L} \\
\mathrm{~L}-1.0 \mathrm{EQX}-0.3 \mathrm{EQY}\end{array}$ & & DX & -1. \\
\hline $\begin{array}{l}1.34 \mathrm{SDL}+1.34 \mathrm{DL}+0.5 \mathrm{~L} \\
\mathrm{~L}+0.3 \mathrm{EQX}+1 \mathrm{EQY}\end{array}$ & & DY & 1. & $\begin{array}{l}\text { 1.34SDL+1.34DL+0.5L } \\
\mathrm{L}-1.0 \mathrm{EQX}-0.3 \mathrm{EQY}\end{array}$ & & DY & -0.3 \\
\hline 1.34SDL+1.34DL+0.5L & Linear Add & DEAD & 1.34 & $\begin{array}{l}\text { 1.34SDL+1.34DL+0.5L } \\
\mathrm{L}+1.0 \mathrm{EQX}-0.3 \mathrm{EQY}\end{array}$ & Linear Add & DEAD & 1.34 \\
\hline$\frac{\mathrm{L}-0.3 \mathrm{EQX}-1 \mathrm{EQY}}{1.34 \mathrm{SDL}+1.34 \mathrm{DL}+0.5 \mathrm{~L}}$ & Lilleâl Aud & DEAD & 1.34 & $\begin{array}{l}1.34 \mathrm{SDL}+1.34 \mathrm{DL}+0.5 \mathrm{~L} \\
\mathrm{~L}+1.0 \mathrm{EQX}-0.3 \mathrm{EQY}\end{array}$ & & SUPERDEAD & 1.34 \\
\hline L-0.3EQX-1EQY & & SUPERDEAD & 1.34 & $\begin{array}{l}\text { 1.34SDL+1.34DL+0.5L } \\
\mathrm{L}+1.0 \mathrm{EQX}-0.3 \mathrm{EQY}\end{array}$ & & Live & 0.5 \\
\hline $\begin{array}{l}\text { 1.34SDL+1.34DL+0.5L } \\
\text { L-0.3EQX-1EQY }\end{array}$ & & Live & 0.5 & \begin{tabular}{|l}
$\mathrm{L}+1.0 \mathrm{EQX}-0.3 \mathrm{EQY}$ \\
$1.34 \mathrm{SDL}+1.34 \mathrm{DL}+0.5 \mathrm{~L}$ \\
$\mathrm{~L}+1.0 \mathrm{EQX}-0.3 \mathrm{EQY}$
\end{tabular} & & DX & 1. \\
\hline $\begin{array}{l}\text { 1.34SDL+1.34DL+0.5L } \\
\mathrm{L}-0.3 \mathrm{EQX}-1 \mathrm{EQY}\end{array}$ & & DX & -0.3 & $\begin{array}{l}1.34 \mathrm{SDL}+1.34 \mathrm{DL}+0.5 \mathrm{~L} \\
\mathrm{~L}+1.0 \mathrm{EQX}-0.3 \mathrm{EQY}\end{array}$ & & DY & -0.3 \\
\hline $\begin{array}{l}\text { 1.34SDL+1.34DL+0.5L } \\
\text { L-0.3EQX-1EQY }\end{array}$ & & DY & -1. & $\begin{array}{l}\text { 1.34SDL+1.34DL+0.5L } \\
\mathrm{L}-1.0 \mathrm{EQX}+0.3 \mathrm{EQY} \\
\end{array}$ & Linear Add & DEAD & 1.34 \\
\hline $1.34 \mathrm{SDL}+1.34 \mathrm{DL}+0.5 \mathrm{~L}$ & $\mathrm{~J}$ inor $A d d$ & DFAD & 124 & $\begin{array}{l}\text { 1.34SDL+1.34DL+0.5L } \\
\mathrm{L}-1.0 \mathrm{EQX}+0.3 \mathrm{EQY}\end{array}$ & & SUPERDEAD & 1.34 \\
\hline $\mathrm{L}+0.3 \mathrm{EQX}-1 \mathrm{EQY}$ & Linear Add & DEAD & 1.34 & $1.34 \mathrm{SDL}+1.34 \mathrm{DL}+0.5 \mathrm{~L}$ & & I ive & 05 \\
\hline $1.34 \mathrm{SDL}+1.34 \mathrm{DL}+0.5 \mathrm{~L}$ & & & & $\mathrm{~L}-1.0 \mathrm{EQX}+0.3 \mathrm{EQY}$ & & & \\
\hline $\mathrm{L}+0.3 \mathrm{EQX}-1 \mathrm{EQY}$ & & SUPERDEAD & 1.34 & $\begin{array}{l}1.34 \mathrm{SDL}+1.34 \mathrm{DL}+0.5 \mathrm{~L} \\
\mathrm{~L}-1.0 \mathrm{EQX}+0.3 \mathrm{EQY}\end{array}$ & & DX & -1. \\
\hline $\begin{array}{l}1.34 \mathrm{SDL}+1.34 \mathrm{DL}+0.5 \mathrm{~L} \\
\mathrm{~L}+0.3 \mathrm{EQX}-1 \mathrm{EQY}\end{array}$ & & Live & 0.5 & $\begin{array}{l}1.34 \mathrm{SDL}+1.34 \mathrm{DL}+0.5 \mathrm{~L} \\
\mathrm{~L}-1.0 \mathrm{EQX}+0.3 \mathrm{EQY}\end{array}$ & & DY & 0.3 \\
\hline
\end{tabular}

This work is licensed under a Creative Commons Attribution-ShareAlike 4.0 International License. 
E-ISSN: 2685-3272 | P-ISSN 1412-0860

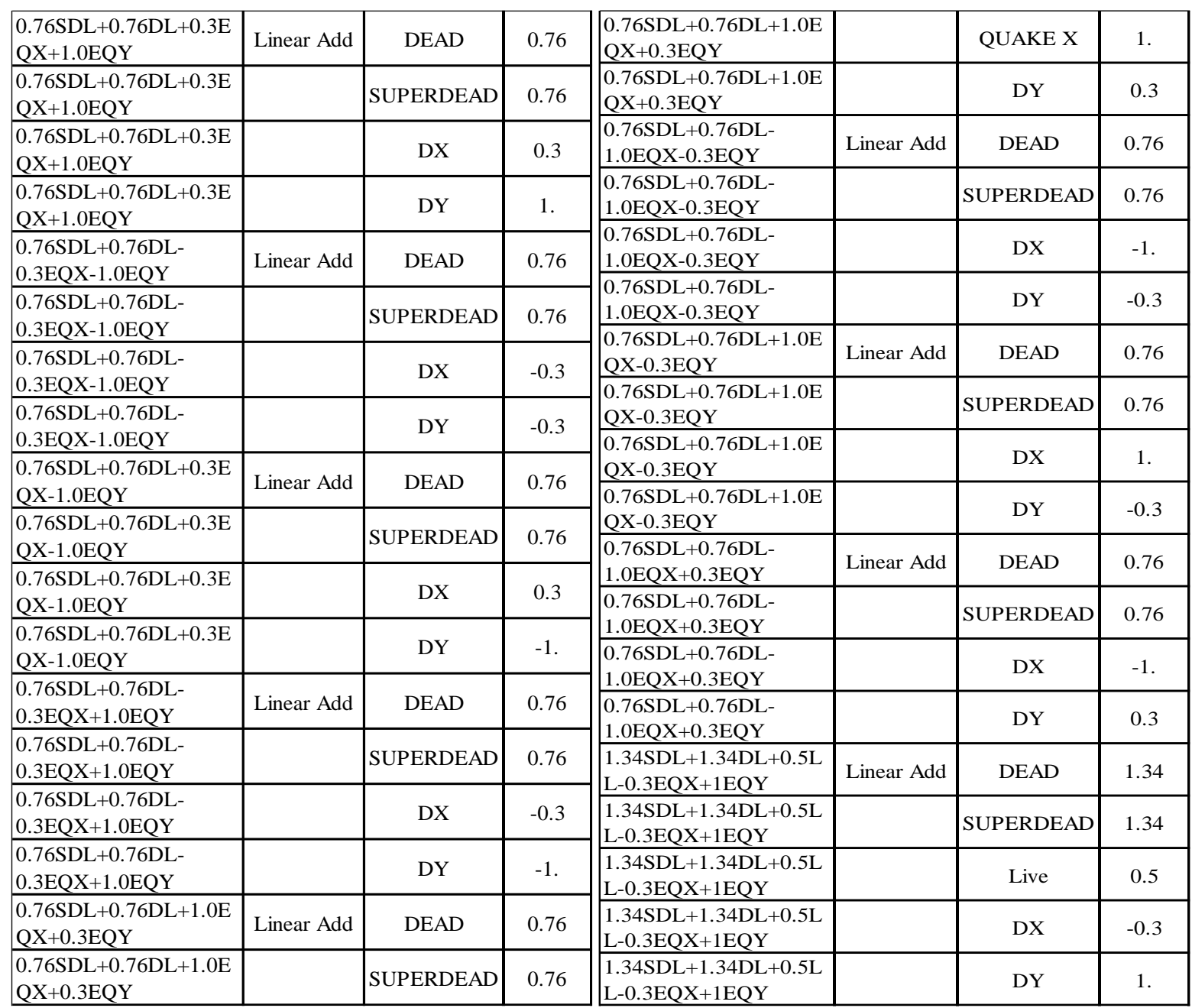

\subsection{Results of Analysis Static}

The results from the SAP2000 v21Avizan \& Koespiadi, (2020) program, the floor shear force due to dynamic earthquake loads is generated by inputting the seismic response spectrum used based on the earthquake zone used. The following is a graph of the SAP2000 v21 input spectrum response.

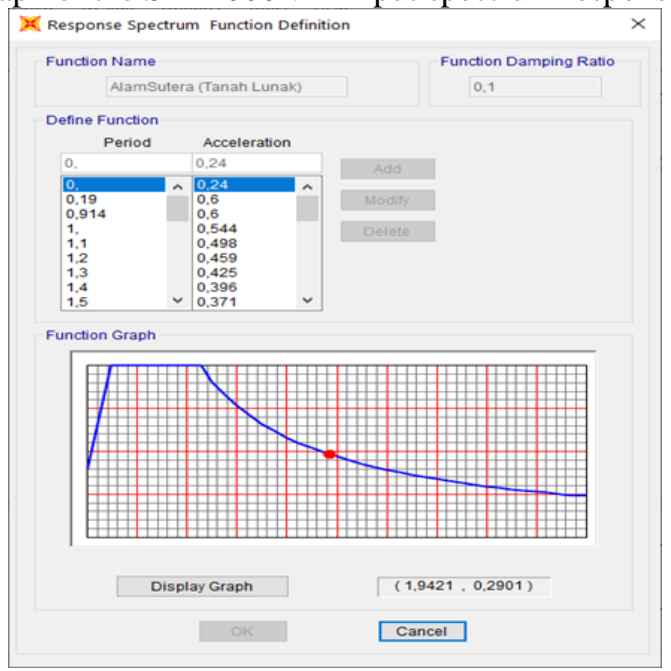

Figure 13. Density Change of Concrete Samples $\left(\mathrm{kg} / \mathrm{m}^{3}\right)$

This work is licensed under a Creative Commons Attribution-ShareAlike 4.0 International License. 
Table 17. Response Spectrum SkyHouse Alam Sutera

\begin{tabular}{|c|c|c|c|c|c|c|}
\hline case & Load type & Load name & Coord sys & funtion & $\begin{array}{c}\text { Angle } \\
\text { degrees }\end{array}$ & $\begin{array}{c}\text { Tran AccSF } \\
\mathrm{mm} / \mathrm{sec} 2\end{array}$ \\
\hline DX & Acceleration & U1 & GLOBAL & $\begin{array}{l}\text { Alam } \\
\text { Sutera }\end{array}$ & 0. & 1226.25 \\
\hline DY & Acceleration & $\mathrm{U} 2$ & GLOBAL & $\begin{array}{l}\text { Alam } \\
\text { Sutera }\end{array}$ & 0 . & 1226.3 \\
\hline QUAKE X & Acceleration & U1 & GLOBAL & $\begin{array}{l}\text { Alam } \\
\text { Sutera }\end{array}$ & 0 . & 1226.25 \\
\hline QUAKE Y & Acceleration & $\mathrm{U} 2$ & GLOBAL & $\begin{array}{l}\text { Alam } \\
\text { Sutera }\end{array}$ & 0 . & 1226.3 \\
\hline
\end{tabular}

Table 18.. Dynamic Earthquake Response Based on SAP2000 V21 . Results

\begin{tabular}{|c|c|c|c|c|c|c|c|}
\hline OutputCase & StepType & GlobalFX & GlobalFY & GlobalFZ & GlobalMX & GlobalMY & GlobalMz \\
\hline Text & Text & $\mathrm{KN}$ & $\mathrm{KN}$ & $\mathrm{KN}$ & $\mathrm{KN}-\mathrm{m}$ & $\mathrm{KN}-\mathrm{m}$ & $\mathrm{KN}-\mathrm{m}$ \\
\hline MODAL & Mode & 0,04 & $-0,04$ & 0,00 & 1,30 & $-4,77$ & $-1,06$ \\
\hline MODAL & Mode & $-0,02$ & $-0,04$ & 0,00 & 6,11 & 2,18 & $-1,06$ \\
\hline MODAL & Mode & 0,00 & 0,08 & 0,00 & $-2,95$ & $-0,40$ & $-0,57$ \\
\hline MODAL & Mode & 0,00 & 0,00 & $-0,01$ & $-0,11$ & 0,30 & 0,20 \\
\hline MODAL & Mode & 0,19 & $-0,06$ & $-0,01$ & $-1,21$ & $-4,70$ & $-4,50$ \\
\hline MODAL & Mode & 0,00 & 0,00 & $-0,02$ & $-0,33$ & 0,01 & $-0,03$ \\
\hline MODAL & Mode & $-0,06$ & $-0,21$ & 0,02 & 1,15 & 0,52 & $-5,32$ \\
\hline MODAL & Mode & $-0,01$ & 0,00 & 0,02 & 0,38 & $-0,05$ & 0,13 \\
\hline MODAL & Mode & $-0,01$ & 0,01 & $-0,05$ & $-0,98$ & 1,86 & 0,43 \\
\hline MODAL & Mode & 0,00 & $-0,01$ & $-0,02$ & $-0,07$ & 0,82 & $-0,36$ \\
\hline MODAL & Mode & 0,03 & $-0,39$ & 0,02 & 6,65 & 0,54 & 3,33 \\
\hline MODAL & Mode & 0,00 & $-0,01$ & 0,03 & 0,51 & $-0,01$ & $-0,06$ \\
\hline SUPERDEAD & & 9618,34 & $-153662,91$ & 3850049,57 & 72629247,20 & $-101615971,00$ & $-2566202,52$ \\
\hline DX & Max & 9207,20 & 6323,26 & 248,40 & 297282,26 & 888095,30 & 206368,46 \\
\hline DY & Max & 3175,18 & 8380,25 & 451,65 & 831265,92 & 332334,09 & 201556,47 \\
\hline QUAKE X & Max & 9206,82 & 6323,00 & 248,39 & 297270,14 & 888059,09 & 206360,05 \\
\hline QUAKE Y & Max & 3175,18 & 8380,25 & 451,65 & 831265,92 & 332334,09 & 201556,47 \\
\hline
\end{tabular}

Table 19. Earthquake Period Based on SAP2000 V21 Results

\begin{tabular}{|l|l|l|l|l|l|l|}
\hline $\begin{array}{l}\text { Output } \\
\text { Case }\end{array}$ & $\begin{array}{l}\text { Step } \\
\text { Type }\end{array}$ & Step Num & Period & Frequency & CircFreq & Eigenvalue \\
\hline Text & Text & Unitless & Sec & Cyc/sec & rad/sec & rad2/sec2 \\
\hline MODAL & Mode & 1 & 19,49909 & 0,051284 & 0,32223 & 0,10383199 \\
\hline MODAL & Mode & 2 & 16,87373 & 0,059264 & 0,372365 & 0,13865567 \\
\hline MODAL & Mode & 3 & 13,41779 & 0,074528 & 0,468273 & 0,21927939 \\
\hline MODAL & Mode & 4 & 6,943229 & 0,144025 & 0,904937 & 0,81891103 \\
\hline MODAL & Mode & 5 & 5,919126 & 0,168944 & 1,061506 & 1,12679422 \\
\hline MODAL & Mode & 6 & 4,939493 & 0,20245 & 1,272031 & 1,61806163 \\
\hline MODAL & Mode & 7 & 4,858961 & 0,205805 & 1,293113 & 1,67214096 \\
\hline MODAL & Mode & 8 & 4,416993 & 0,226398 & 1,422503 & 2,02351411 \\
\hline MODAL & Mode & 9 & 4,200074 & 0,238091 & 1,49597 & 2,23792645 \\
\hline MODAL & Mode & 10 & 3,97254 & 0,251728 & 1,581654 & 2,50163061 \\
\hline MODAL & Mode & 11 & 3,578329 & 0,27946 & 1,755899 & 3,08318161 \\
\hline MODAL & Mode & 12 & 3,418921 & 0,29249 & 1,837769 & 3,37739306 \\
\hline Modus Min: & 19,49909 & 0,29249 & 1,837769 & 3,37739306 \\
\hline Modus Max: & 3,418921 & 0,051284 & 0,32223 & 0,10383199 \\
\hline
\end{tabular}


E-ISSN: 2685-3272 | P-ISSN 1412-0860

Table 20. Modal Participation Factors

\begin{tabular}{|c|c|c|c|c|c|c|c|c|c|c|c|c|c|c|c|}
\hline ttputcas & & & Period & UX & UY & UZ & SumUX & SumUY & SumU2 & RX & RY & RZ & SumRX & SumRY & SumRZ \\
\hline Text & Text & Unitless & Sec & Unitless & Unitless & Unitless & Unitless & Unitless & Unitless & Unitless & nitless & Unitless & Unitless & Unitless & Wnittoce \\
\hline DAL & I & & 19,499087 & 0,59446 & 0,06909 & 0,00001845 & 0,59446 & & 0,00001845 & 3465 & 9144 & 00317 & 33465 & ,29144 & \\
\hline DAL & & & 16,873728 & 0,06741 & & & & & 0,00007079 & & & & 29781 & 0,32592 & \\
\hline DAL & & & 13,417789 & 0,00089 & 0,05051 & 0,00003582 & 0,66276 & 0089 & 0,00011 & & & 58756 & 4809 & 22625 & \\
\hline DAL & & & 6,943229 & $5,274 E-11$ & $4,673 E-07$ & 0,00018 & 0,66276 & 0,6809 & 0,00028 & & & & 0,3481 & 0,32627 & \\
\hline DAL & & & 5,919126 & 0,14896 & 0,00614 & 0,00008868 & & & & & & & & $0,1]$ & \\
\hline DAL & & 0 & 4,939493 & $7,2955-09$ & $6,105 E-08$ & 0,00022 & 0,81173 & 0,68704 & & & 0,00011 & $5,784 E-08$ & 0,36926 & 0,51511 & \\
\hline DAL & INoue & & 4,858961 & 0,01079 & 0,10876 & 0,00029 & 0,82252 & 0,7958 & 0,00089 & 0,24769 & 0,01888 & 0,00776 & 0,61695 & 0,53399 & \\
\hline DAL & Mode & 0 & 4,416993 & $6,338 E-08$ & 0,000001161 & 0,00016 & 0,82252 & 0,7958 & 0,00105 & 0,00001169 & 0,00007062 & $5,177 \mathrm{E}-08$ & 0,61696 & \begin{tabular}{|l|}
0,53406 \\
\end{tabular} & \\
\hline DAL & Mode & 0 & 4,200074 & $1,908 E-08$ & $3,781 E-08$ & 0,00083 & 0,82252 & 0,7958 & 0,00187 & 0,00002259 & 0,00012 & $5,184 E-09$ & 0,61699 & $\begin{array}{l}0,53418 \\
\end{array}$ & \\
\hline DAL & $4 d$ & 10 & 3,97254 & $1,1477-07$ & $6,959 E-07$ & 0,00013 & 0,82252 & 0,7958 & 0,002 & 0,00003362 & 0,00002323 & 0,00000137 & 0,61702 & 0,5342 & \\
\hline DAL & inver & 1 & 3,578329 & 0,00039 & 0,01444 & 0,00011 & 0,82291 & 0,81024 & 0,00212 & 0,00872 & 0,00198 & 0,14158 & 0,02573 & 0,53619 & \\
\hline DAL & Mode & 12 & 3,418921 & $4,811 E-08$ & $5,469 \mathrm{E}-07$ & 0,00019 & 0,82291 & 0,81024 & 0,00231 & 0,000001773 & 0,0001 & 0,0000107 & 0,02574 & 0,53629 & \\
\hline & & & 19,499087 & 0,59446 & 0,5613 & 0,00083 & 0,82291 & 0,81024 & 0,00231 & 0,26316 & 0,29144 & 0,58756 & 0,02574 & 0,53629 & \\
\hline & & odus Max: & 3,418921 & $5,274 E-11$ & $3,781 E-08$ & 0,00001845 & 0,59446 & 0,06909 & 0,00001845 & $7,7466-07$ & 0,00001928 & $5,184=-09$ & 0,03465 & 0,29144 & \\
\hline
\end{tabular}

\section{Conclusion}

After analyzing using the response spectrum method, the authors can draw the following conclusions based on the results of the SAP2000 v21 program.

1. comparison of the upper structure between planning data and modification data (For Construction) The results of the maximum displacement in the $\mathrm{X}$ direction are $0.02373 \mathrm{~m}$ and the $\mathrm{Y}$ direction is $0.181128 \mathrm{~m}$ so that it still meets the maximum limit $[(0.015 \mathrm{hsx}) / \mathrm{\rho}]=1.662 \mathrm{~m}$

2. Because the displacement and base shear results in the equivalent lateral force analysis are greater than in the variance response spectrum analysis, the results of the equivalent lateral force analysis are safer if used for the application of earthquake force loading in structural calculations.

3. The result of the comparison between the calculation of the nominal static shear force and the nominal dynamic shear analysis is greater.

\section{References}

Avizan, A. B., \& Koespiadi, K. (2020). Comparison of Building Structure Planning with SNI 2847-2013 Method and SAP2000 V.19 Software Code ACI 318-14. IJTI (International Journal of Transportation and Infrastructure), 3(1), 1-10. https://doi.org/10.29138/ijti.v3i1.1050

B. \& Wungo, W. C. (2012). Analisis beban gempa statik ekivalen.

SNI 03-2847-2002. (n.d.). "Pedoman Perhitungan Struktur Beton untuk Bangunan Gedung."

\section{Biography}

\section{Agus Fernando}

I am Agus Fernando, born in Jakarta, August 10, 1992. I started studying at Mercubuana University in 2016/2017 Now I work at the North Jakarta Administration of Water Resources Sub-dept. as PPJU. My work experience was mostly in engineering before I worked at the Water Resources Sub-Department, I worked as an Engineer for several projects including Structural Engineering at PT. Hyundai Aluminum Internasional, Head Engineer at PT. AluMakro Korea, Structural Draftsman at PT. Wijaya Karya and many more of my work experiences.

\section{Syafwandi}

This work is licensed under a Creative Commons Attribution-ShareAlike 4.0 International License. 
He was born in Jakarta, October 13, 1956. As Professor at UMB, Bachelors Degree at University Indonesia (1984), Masters degree at Instute Teknologi Bandung and University college London, 1988 and finish S3 as doctor at University Satyagama (2005).

\section{Agung Sumarno}

He was born Samarinda, July 5, 1990. Teaching at the Civil Engineering undergraduate program, Univ. Mercubuana Bachelors Degree at Univ. Mulawarman Samarinda, Engineering faculty majoring in civil engineering, Masters degree at Bandung Institute of Technology, Faculty of Civil and Environmental Engineering majoring in Civil Engineering Structural Engineering. And He also as a Researcher at the LIPI, biomaterials research center.

\section{Resi Aseanto}

He was born Cilacap, June 23, 1980. Teaching at the Civil Engineering undergraduate program, Univ. Mercubuana Bachelors Degree at Diponegoro University, Faculty of Civil Engineering, majoring in Civil Engineering, Masters degree at University of Indonesia majoring in Engineering, Master in Management IPMI International Business School, Gajahmada University Engineering Profession Program. And He also as Served as Director of Operations \& Commercial and concurrently as Acting. President Director at PGNMAS. He also had many work experience including Kalija Project as Procurement and Contract Manager (2014), CP 16 Project as Procurement and Contract Manager (2015), Commercial General Manager of PT PGAS Solution (2016), Head of Business Development and Risk Management Division and Performance Control PT PGAS Solution (2017) 